\title{
Obesity Prevention and Management across the Lifespan
}

\author{
Christy Barnhart \\ California University of Pennsylvania, California, PA, USA \\ Email: cjbarnhart@hsc.wvu.edu
}

How to cite this paper: Barnhart, C. (2020) Obesity Prevention and Management across the Lifespan. Open Access Library Journal, 7: e6733.

https://doi.org/10.4236/oalib.1106733

Received: August 21, 2020

Accepted: October 27, 2020

Published: October 30, 2020

Copyright $\odot 2020$ by author(s) and Open Access Library Inc.

This work is licensed under the Creative Commons Attribution International License (CC BY 4.0).

http://creativecommons.org/licenses/by/4.0/ (c) (i) Open Access

\begin{abstract}
Obesity is a disease that can be prevented or managed for individuals across the lifespan. Individuals can modify diet and increase physical activity to improve health outcomes and improve quality of life. Individuals who use these modification strategies to improve health can further prevent other chronic illnesses affiliated with obesity from occurring. The alternative to being proactive or reactive to obesity prevention or management would be the development of associated chronic illnesses, which can result in a shortened lifespan. Healthcare providers share a common vision of educating individuals regarding diet modification and incorporating exercise in the daily routine to prevent obesity, which is an important task for educators as well to make a difference in populations of all ages. Healthcare workers and educators who deliver information to populations in need, gain trust and cooperation by treating others using the guiding principles of dignity, honesty, and integrity which can produce mutual respect, build rapport, and create trusting relationships that can lead to compliance of the plan for obesity prevention or management. Continuing education for obesity prevention, including diet and exercise, can produce positive outcomes in the community and result in lifestyle modifications for healthy behaviors for individuals of all ages.
\end{abstract}

\section{Subject Areas}

Nursing

\section{Keywords}

Obesity, Prevention, Management, Diet, and Exercise

\section{Introduction}

\subsection{Defining Obesity, Signs, and Symptoms}

Obesity is a complex disease that affects people of all ages. Defining obesity will 
bring clarity to the problem. Reviewing signs and symptoms of obesity will help to identify individuals in need of support. The information regarding obesity can potentially assist individuals with obesity prevention or management, regarding obesity or other chronic illnesses affiliated with obesity. Obesity will be defined in the pediatric and adolescent (child) population as well as adults. The provided information will enhance knowledge of obesity and explore the various populations in need, as well as methods used to prevent, manage or treat obesity (CDC, 2020 [1]; Mayo Clinic, 2020 [2]; National Institutes of Health (NIH), 2015 [3]).

\subsection{Obesity Defined in Children}

Obesity is diagnosed by using the body mass index (BMI) chart for children along with the pediatric growth chart, which requires the following information: age, gender, height, and weight. A percentile is retrieved after plotting the information on the chart, and an interpretation is made by comparing the percentile to other children matching the age and gender. The interpretation of the findings is as follows: BMI between the 85th and 94th percentile is considered overweight, and a BMI at the 95th percentile or higher is considered obese (CDC, 2020) [1]. The CDC (2020) has growth pediatric charts separated by gender, age, length or height, and weight. For example, there are charts for boys and girls from birth to 36 months. There are also charts for children aged 2 years to 20 years (CDC, 2020) [1].

\subsection{Obesity Defined for Adults}

Obesity is a disease that is defined as having an excessive amount of body fat (CDC, 2020 [1]; Mayo Clinic, 2020 [2]; NIH, 2019 [4]). Obesity places individuals at risk for heart disease, diabetes, high blood pressure, and certain cancers. Obesity is diagnosed when an individual has a BMI of 30 or higher. BMI is a calculation derived from an adult's height in feet and inches, and weight in pounds. A reference chart for BMI parameters in the adult is as follows (Mayo Clinic, 2020) [2]:

\begin{tabular}{cc}
\hline BMI & Weight Classification \\
\hline Below 18.5 & Underweight \\
$18.5-24.9$ & Normal \\
$25.0-29.9$ & Overweight \\
30.0 and higher & Obesity \\
\hline
\end{tabular}

According to Mayo Clinic (2020) [2], a diagnosis for obesity can be obtained by having the following: a health history completed, a physical exam, and obtaining diagnostic information. In addition to having a BMI of 30 or greater, the following diagnostic test can be diagnostic for obesity: a waist circumference of more than 35 inches for females, waist circumference of more than 40 inches for males, and blood work to establish: cholesterol levels, liver functions, fasting 
glucose, and thyroid testing. These diagnostic tests can give healthcare providers an in-depth look at individual health status regarding obesity and affiliated chronic illnesses. A chart is included below to show the normal values of diagnostic tests, and obesity would be considered for values above the normal levels (NIH, 2015) [3]:

\begin{tabular}{cc}
\hline Test & Normal Values \\
\hline BMI & $18.5-24.9$ \\
Waist Size & Men $<40$ ”/Women $<35$ ” \\
Blood Pressure & $120 / 80 \mathrm{~mm} \mathrm{Hg}$ or less \\
LDL-Bad Cholesterol & Less than $100 \mathrm{mg} / \mathrm{dl}$ \\
HDL-Good Cholesterol & Men $>40 \mathrm{mg} / \mathrm{dl} ;$ Women $>50 \mathrm{mg} / \mathrm{dl}$ \\
Triglycerides & Less than $150 \mathrm{mg} / \mathrm{dl}$ \\
Fasting Blood Glucose & Less than $100 \mathrm{mg} / \mathrm{dl}$ \\
\hline
\end{tabular}

\subsection{Causes and Risk Factors of Obesity}

Obesity is caused by taking in an excessive number of calories compared to the number of calories being burned, which is a modifiable cause (CDC, 2020 [1]; Mayo Clinic, 2020 [2]; NIH, 2015 [3]). This leads to storage of unused energy in the form of fat. Limiting the amount of high calorie and unhealthy foods can help individuals prevent obesity and decrease the risk of other chronic illnesses affiliated with obesity.

Risks associated with obesity include type 2 diabetes, high blood pressure, heart disease, stroke, fatty liver disease, and kidney disease. These chronic illnesses affect almost every system within the human body and can lead to a shortened lifespan NIH (2019) [4]. Type 2 diabetes is caused by limited activity levels, poor diet, and body fat, especially in the abdominal area. High blood pressure or hypertension can lead to kidney problems, heart disease, or stroke. Being obese causes strain on the heart, creating a need for the heart to work harder; therefore, causing pressure within the vessels and hypertension. Due to obesity, or hypertension, the increased wear and tear on the pump of the heart can cause a fatal cardiac event to occur, such as myocardial infarction (heart attack). The heart can also go into other arrhythmias, such as atrial fibrillation, which can lead to a stroke. Fatty liver disease is a risk of being obese and results from a buildup of fat in the liver. If obesity is not managed for individuals with fatty liver disease, the risk is non-alcohol-related cirrhosis of the liver (NIH, 2019) [4].

\subsection{Obesity Complications}

Obesity complications involve development of obesity or comorbidity with other chronic illnesses such as heart disease, diabetes, high blood pressure, and certain cancers. Heart disease negatively affects blood pressure and cholesterol, which places obese individuals at risk of cardiac events as well as stroke. Type 2 di- 
abetes is another disease that has the potential of effecting obese individuals related to insulin resistance. Type 2 diabetes can impair wound healing, and over time can negatively affect the heart, kidneys, and the blood vessels. Certain cancers, such as: cervical, ovarian, breast, colon, and esophageal, are other complications of obesity that can negatively affect obese individuals. Other affiliated health complications related to obesity can include digestive issues, gynecological or sexual problems, sleep apnea, and osteoarthritis (CDC, 2020 [1]; Mayo Clinic, 2020 [2]; NIH, 2015 [3]).

\subsection{Obesity Prevention or Treatment}

To prevent obesity, incorporation of diet and exercise is essential to prevent obesity and to manage or treat obesity. According to Harada et al. (2018) [5], 150 minutes of moderate exercise weekly can help prevent obesity and manage weight. According to ChooseMyPlate (https://www.choosemyplate.gov) [6], the 2015-2020 guidelines recommend following the portion sizes regarding protein, whole grains, fruits, vegetables, and dairy so that individuals can manage daily nutritional intake and lose weight. The pediatric population can benefit from learning how to use portion control and increase physical activity while in school and at home to develop good habits to continue into adulthood.

\subsection{Interventions Strategies Using Levels of Prevention}

Obesity is a chronic disease which is associated with many other chronic diseases that can become life-threatening. The people diagnosed with obesity keep increasing in numbers, and there is a need for prevention methods to be introduced to individuals of all ages.

Introducing the prevention strategies to all individuals can make a difference. The intervention strategies which use the levels of prevention include primary prevention, secondary prevention, and tertiary prevention (CDC, 2011 [7]; Kisling \& Das, 2019 [8]). Primary prevention involves the prevention of disease before the disease becomes a problem. An example of primary prevention is the incorporation of diet and exercise, which are interventions used to prevent someone who is not obese from becoming obese. Secondary prevention involves overweight individuals who can use information regarding methods to manage weight to decrease the risk of developing other chronic illnesses. Secondary prevention also involves diagnostic testing used to diagnose obesity and affiliated chronic illnesses or to monitor the progression of obesity and affiliated chronic illnesses. Tertiary prevention involves preventions which are utilized by individuals who are already obese and have affiliated chronic illnesses (Hoelscher et al., 2015) [9]. The treatment plan for the various levels of prevention may slightly differ. For example, diet and exercise are the ideal treatment plan for primary, secondary, and tertiary prevention strategies, but with tertiary prevention, there may be an additional treatment needed to treat the other chronic illness affiliated with obesity (Hoelscher et al., 2015) [9]. The prevention interven- 
tion strategies included in this paper involve adding nutrition and increased physical activity into the equation to improve health, prevent other chronic diseases affiliated with obesity, and manage or treat obesity and weight for all individuals. Across the lifespan, the pediatric to adolescent age group can benefit from obesity prevention strategies with nutrition education by making modifications in diet and incorporation of increased physical activity with support from the community (school) or in the home (parental support).

An evaluation of the resources used to provide a foundational meaning on the topic of obesity prevention and management, the websites and articles used to support a need to incorporate prevention efforts for all obese individuals. Identifying individuals at risk can provide healthcare providers with enough time to develop a plan or strategy. Utilizing diagnostic information can lead to treatments such as improved nutrition and increased physical activity (CDC, 2011 [7]; CDC, 2020 [1]; Harada et al., 2018 [5]; Hoelscher et al., 2015 [9]; Kisling \& Das, 2019 [8]; Mayo Clinic, 2020 [2]; NIH, 2015 [3]; NIH, 2019 [4]).

\section{Pediatric and Adolescent}

\subsection{Obesity Factors That Contribute to Obesity}

Factors that contribute to obesity include low-socioeconomic status, ethnic considerations, and other chronic illnesses (Cloutier et al., 2018 [10]; French et al., 2018 [11]; Gatto et al., 2017 [12]; Hull et al., 2018 [13]; Lofton et al., 2016 [14]; Smith et al., 2016 [15]; Trude et al., 2018 [16]; Woodward et al., 2018 [17]). The relationship between low-socioeconomic status and ethnicity include negative influences that affect these factors such limited access to healthy food options due to expense and durability of food, places to engage in physical activity, and physical limitations present from the presence of another chronic illness. Available programs to support these populations with opportunities to receive help in obtaining healthier food options and safe places for children to play or engage in physical activity can prevent childhood obesity and future chronic disease (Mayo Clinic,2020) [2].

\subsection{Low-Socioeconomic Status}

Smith et al. (2016) [15] used a randomized control trial to see if resistance training could benefit adolescents by improving muscular fitness. This research involved teenage boys, who were participating in the Active Teen Leaders Avoiding Screen Time. The participants in this study were recruited from low-socioeconomic families who were at risk for obesity because of the limited access to healthy foods and physical activity. The study looked at body fat percentage, muscular fitness, physical activity, and resistance training skills prior to intervention and eight months later for post-intervention data. This study identified a way to improve body composition and muscular fitness among adolescent males who are in the low-socioeconomic status. The results of this study showed that resistance training could help to improve body composition and muscular fitness among 
adolescent males who are in the low-socioeconomic status and that resistance training could help to improve body composition and muscular growth in teenage boys. This research supports obesity prevention and management using resistance training in low-socioeconomic status teenage boys.

An Early Childhood Obesity Prevention Program (ECHO), was developed by Cloutier et al. (2018) [10] to help moms develop obesity prevention strategies for their babies. This pilot, randomized control trial studied disadvantaged moms with newborn babies who were recruited when pregnant or just delivering a baby. Moms were asked to set goals for obesity prevention and develop a strategy. Topics regarding the consumption of juice, sugar-sweetened beverages, solids, breastfeeding, infant sleeping patterns, and television time were discussed with the moms. The participants were recruited from a low-income neighborhood in Hartford, CT; this study included moms and babies. The study looked at moms who were enrolled in this program and received the nurturing family network information regarding health and wellness strategies for moms and babies, such as healthy eating habits and physical activity. The results of the study showed that the participants used coupons to purchase healthier options in the grocery store (Cloutier et al., 2018) [10]. This article by Cloutier et al. (2018) [10], was an excellent resource on the topic of obesity prevention in the pediatric population and supports research for individuals in a low-socioeconomic group who can benefit from having access to healthier food choices and prevent obesity.

A multi-component obesity prevention intervention involving community-based parenting classes can prevent obesity in low-income preschoolers (French et al., 2018) [11]. This randomized control trial recruited 534 children from multiple primary care clinics in Minneapolis who were between the ages of 2 - 4 . There were 269 children who were in the control group, and 265 children were in the intervention group. The intervention involved teaching parents how to prepare healthy food for their children and how to increase physical activity to prevent obesity.

The study looked at body mass index, dietary intake, and physical activity of the participants over three years of age. The results of the study showed that the children in the intervention group were able to decrease the BMI, increase physical activity levels, and improve dietary intake by decreasing the amount of sugar consumed. This study supported a need for the prevention of obesity for children in low-income families by teaching parents how to prepare healthy meals.

There are many reasons that children have limited access to healthy foods that prevent obesity, such as: race, ethnicity, region, and socio-economic status (Woodward et al., 2018) [17]. This observational study with randomization recruited 5138 children in two elementary schools and middle schools in 130 communities in the United States between 2013-2015. The study reviewed the dietary intake of sugar, energy-dense foods, fresh vegetables, whole grains, and dietary fiber. Other factors that were reviewed were: age, race, ethnicity, gender, annual income, maximum parental education, and employment status. The re- 
sults of the study showed that obesity is linked to poor dietary intake, which is an effect of eating unhealthily and related to low-socioeconomic status. This study shows moderate evidence that supports a need for behavioral change toward dietary habits within low-socioeconomic population to positively affect prevention of childhood obesity.

Low-socioeconomic status is a factor that has a negative effect on pediatric obesity, and the research studies presented provided a need to assist this population change lifestyle habits. Introducing resistance training to teenage boys can have a positive impact on obesity prevention for low-income adolescents. New moms can start engaging in healthy habits from the start, and with support can provide healthier food options for disadvantaged children. Community-based classes can assist low-income families to learn how to prepare healthier meals in the home.

These articles present information to support obesity prevention strategies within the community and home for children living within a low-income family and have limited opportunities and resources (Cloutier et al., 2018 [10]; French et al., 2018 [11]; Smith et al., 2016 [15]; Woodward et al., 2018 [17]).

\subsection{Ethnic Considerations}

A cluster-randomized trial design reviewed a family-based weight gain prevention intervention regarding the Hispanic population (Hull et al., 2018) [13]. The 206 participants were recruited from a metropolitan area in Nashville, Tennessee. Individuals who were involved in the study included one parent or guardian per family and one eligible child between the ages of $5-7$. The participants were broken into two groups: the intervention group, which focused on weight gain prevention, and the control group, which focused on oral health. Some variables that were reviewed included BMI, waist circumference, physical activity level, dietary behaviors, and dietary preferences. The results of the study showed that at least 24 contact hours of physical activity is needed in order to see a decrease in weight. The results also showed a correlation between time spent exercising with an increased amount of weight loss. This study provides quality information that supports options for the Hispanic population to prevent obesity by increasing activity levels and modifying diet.

An article by Gatto et al. (2017) [12], reviewed diverse populations residing in the Los Angeles area and involved in a gardening program to reduce the risk of obesity in this population. This randomized control trial had an intervention group and a control group. The intervention group involved 172 third to fifth graders, and the control group involved 147 third to fifth graders. The control groups involved students from two different schools who did not have a gardening program. The participants had to be Hispanic or Latino and from Los Angeles. The study reviewed the participant's dietary intake, BMI, waist circumference, and fasting lab studies. The classes were taught for 90 minutes one day per week for twelve weeks. The results of the study showed that the intervention 
group had positive results when compared to the control group. The study indicated that more time and larger numbers of participants would be ideal in the future. The article by Gatto et al. (2017) [12] is an excellent resource and provides a need to support obesity prevention for diverse populations to use a gardening program in the school setting as way to eat healthier.

A diverse population that was reviewed was the African American youth, who are also from low-income families. This study by Trude et al. (2018) [16], looked at methods to reduce snack consumption among this population. There are many reasons that ethnic populations have limited access to healthy food choices as well as places to exercise. Living in urban areas produces a challenge for individuals to exercise. Another reason could be a lack of finances, and the price of healthy food, as well as the durability of healthy food choices. The participants included 534 individuals who were recruited from 30 low-income areas within the Baltimore area. The participants were between the ages of 9-15. The results of the study showed that there was a decrease in sugar intake in the older population of the sample intervention group when compared to the control group. This article by Trude et al. (2018) [16] is rated as an excellent resource because it is a randomized control trial that was pertinent to pediatric obesity prevention for ethnic populations by reducing sugar intake.

Obesity prevalence is higher in African Americans than in any other ethnic group (Lofton et al., 2016) [14]. Eight online databases were used to obtain information for this systematic review of the literature. The target population was African American youth between the ages of 6 - 18 .

Eleven studies were included in the final review of this study. The author Lofton et al. (2016) [14], determined if the interventions of the studies affected the person, family, or community. The eleven studies represented 1521 participants between the ages of 8 - 17. Interventions involved: physical activity, dietary choices, and psychosocial factors. Results showed that African American youth could benefit from parental involvement in the intervention as well as a mentor to achieve lifestyle changes. The study by Lofton et al. (2016) [14], provided evidence that individuals who are supported can prevent obesity by engaging in physical activity and making dietary changes.

Children who belong to different ethnic groups encounter issues with preventing obesity, which is an issue out of the control for these populations; due to living in urban areas, limited access to healthy foods, or due to cost and durability. Two articles addressed issues within the Hispanic population that had different approaches. One article approached obesity by increasing the amount of physical activity for these children. The other article approached obesity by incorporating a gardening program as a school project to teach students how to eat healthier foods. There were two articles that discussed the African American population and discussed the limited spaces for children to increase physical activity and how having parental involvement with children to offer support and modify lifestyle habits within the home can have positive results in preventing 
obesity. These articles provide evidence to support obesity prevention within these ethnic populations (Hull et al., 2018 [13]; Gatto et al., 2017 [12]; Trude et al., 2018 [16]; Lofton et al., 2016 [14]).

\subsection{Obesity Related to Chronic Illness}

A parent-led physical activity can be used at home with children to increase activity levels. A quasi-randomized trial study by Rubin et al. (2019) [18] involved the use of families with children who were diagnosed with Prader-Willi Syndrome or non-syndromal obesity. According to (NIH, 2020) [4], PWS is a genetic condition that affects many parts of the body. This disease begins in childhood and involves the weakening of the muscles, and in obese children can lead to type 2 diabetes. The participants of this study included 42 kids with PWS and 65 kids with non-syndromal obesity. The participants were between the ages of 1 - 10. The parents received training for use of equipment, and the equipment included outside games to motivate children to engage in physical activity. The study lasted for 24 weeks, and results showed that there was a benefit to having organized physical activity in the home for children with neurologic disorders. In the evaluation of this article by Rubin et al. (2018) [18], a rating that is excellent as this study provides evidence supporting outside games. The evidence indicates that using outside games can motivate children with disabilities to engage in physical activity and prevent obesity as well as type 2 diabetes, which is relevant to the topic of obesity prevention in children with Prader-Willi Syndrome.

Pediatric asthma and obesity are affiliated with each other (Papoutsakis et al., 2018) [19]. The cross-sectional case-controlled study by Papoutsakis et al. (2018) [19] recruited 514 children between the ages of 5 - 11, of which 217 of the children had asthma, and the remainder (control group) were healthy. The research included body mass index calculations, family health history/demographics, dietary assessment, physical activity assessment, and Obesity-preventive lifestyle score (OPLS). The OPLS was developed to determine compliance with lifestyle changes for which a high score was indicative of the likelihood of compliance to a health plan, and a low score indicated that the child would not comply with the plan. The results showed that 158 of the children were obese and that the children who had been diagnosed with asthma had a higher BMI and waist circumference than the children who were in the control group. This study shows evidence that children with asthma are at high risk of becoming obese and need support from family members to prevent obesity.

When evaluating the resources for pediatric obesity in children with chronic illness, there is evidence provided for increased support for children, who have chronic illness, to prevent obesity. The chronic illnesses create a challenge for parents and children to engage in physical activity. Support from schools and healthcare providers may be necessary to prevent or manage obesity in this population. Children with asthma become short of breath if overexerted, and child- 
ren with PWS have muscle weakness. Engaging children in small amounts of physical activity and changing dietary habits can help them live healthier lives (NIH, 2020 [4]; Papoutsakis et al., 2018 [19]; Rubin et al., 2018 [18]).

\subsection{Physical Activity and Nutrition}

There are many prevention strategies or interventions that can be useful in helping children decrease the risk of obesity. Interventions can be provided at school or in the home. Dietary modifications can become manageable with education provided to parents and children to provide additional support and increase knowledge. Increasing physical activity is an important intervention which can help reduce weight and the risk for obesity along with other chronic diseases. Children can maintain health with support from parents and adult role models. Obesity prevention is an important factor when reviewing the pediatric population, and by using a variety of delivery methods to engage and motivate children, successful outcomes can be achieved. Prevention in the school setting can be helpful to school-aged children when prevention can lead to healthy habits to carry into adulthood (CDC, 2019) [20].

\subsection{Use of Games}

Viggiano et al. (2018) [21], used a randomized control trial to study how the board game Kaledo could be used to focus on health promotion by teaching primary school children about portion control. Approximately 1300 students from 10 different schools were recruited and separated into an intervention or control group. The intervention group involved children who played the Kaledo game once per week, and the control group did not participate in playing the game at all. The board game was used to educate students on nutrition and teach students regarding caloric values and increase awareness of dietary intake. Results showed that the participants in the treatment group significantly increased the consumption of healthy foods, decreased the amount of junk food consumed, and decreased body mass index when compared to the control group. This study showed evidence that using an interactive game to teach calorie counting may help to prevent obesity. This research is applicable to obesity prevention in children, as this game can be a useful tool to teach children how to eat healthy foods and portion control.

This randomized control trial by Staiano et al. (2018) [22], looked at new physical activity options for obese children. Forty-six overweight or obese children were randomly assigned to either a control group or an intervention group. The intervention group received a gaming console with exercise games along with a fitness coach to meet with them three times per week for a one-hour session. Children in the control group received exercise games at the final clinic visit. The variables that were measured included BMI, fat mass, and cardiometabolic health metrics. The results of the study showed that the intervention group had a decrease in BMI when compared to the control group over the 
24-week period. The evidence indicates that the intervention of using exercise games to prevent obesity can work.

In the evaluation of these resources, there was evidence supporting community and school-based strategies in the prevention of obesity. Providing opportunities for students to learn how to eat healthier and increase physical activity using games can make losing weight educational and fun. Strategies provided to educate children on how to use portion control when eating by using a game had successful outcomes. These successful outcomes were reflected in the results by increasing consumption of healthy foods, decreasing the intake of high calorie food, decreasing the BMI, and increasing physical activity for the participants (Viggiano et al., 2018) [21]. Fitness coaching, along with a game that incorporated increased physical activity, were reviewed, and participants were able to improve activity levels. The intervention group was able to improve blood pressure, cholesterol, lipid levels, and moderate to vigorous physical activity with $\mathrm{p}$ values $<0.05$ (Staiano et al., 2018) [22]. Both articles provided evidence of support because they were both randomized control trials that took place within the schools and community.

These studies show evidence that teaching children how to eat healthier and utilizing exercise games can decrease BMI (Staiano et al., 2018 [22]; Viggiano et al., 2018 [21]).

\subsection{Home-Based Strategies}

Having support at home is important for a child to develop good habits and successful weight management. According to Byrne et al. (2018) [23], an electronic screening tool can help parents prevent childhood obesity from home. For this randomized control trial, parents of children between the ages of 5-17 were recruited to provide information regarding the height and weight of the children. The screening brief intervention and referral to treatment (SBIRT) tool was used to collect the information. The information that was collected before the study was the child's weight, which was then recorded in the SBIRT and compared to the post weight. The control group was provided with a menu of optional obesity prevention resources. The goal was to inspire the parents to make changes to improve the weight for obese children and change outcomes. Of the 226 parents who participated in the study, results showed that $85 \%$ of the parents were supportive of optional resources available for children to use to prevent or manage childhood obesity. The study results provide evidence that parental involvement in obesity prevention at home can lead to positive outcomes as both participating parents and children can make changes to improve weight.

Well-child visits can have a positive outcome if providers spend time counseling parents and children on dietary behaviors and physical activity (Wright et al., 2018) [24]. This randomized controlled trial consisted of parents with children between the ages of $4-10$. The parents were separated into groups for counseling based on the topic of interest (the delayed group $n=11$ and the imme- 
diate group $n=10$ ): physical activity, fruits and vegetables, television, sweetened drinks, $100 \%$ fruit juice, or no preference. Parents were responsible for reporting the child's physical activity level, television time, and dietary habits. If parents can take responsibility for child activity level and nutritional intake, children could have better outcomes related to obesity prevention. The results of this study showed feasibility, but a larger sample size would be more ideal. The immediate group showed an increase in fruit and vegetable consumption, a decrease in television time, and an increase in physical activity. The article by Wright et al. (2018), shows evidence supporting the topic of obesity prevention, but recommended more time and a larger sample size to determine if parents could take responsibility for reporting the child's physical activity level, television time and dietary habits. Perhaps with the dynamics in families with one parent homes, and working households monitoring the children's behavior would need to involve more people.

Obesity prevention in the home can occur by decreasing the amount of sugar consumed by children. This randomized control trial by Nezami et al. (2018) [25], studied how parents can reduce the chance for children becoming obese from drinking beverages high in sugar content. Participants were recruited by finding moms with a child between the ages of $3-5$. There was a total of 51 participants, of which 27 of the participants were in the intervention group, and 24 of the participants were in the control group. The results of the study revealed that moms in the intervention group lost $2.4 \mathrm{~kg}$ at the 6-month benchmark, and the moms in the control group gained 0.9-lg at the 6-month benchmark. This article provides evidence supporting obesity prevention in children due to parental dietary changes in the home and provides relevant support on the topic of obesity prevention in children.

When evaluating these articles regarding home-based strategies to prevent pediatric obesity, there is evidence for parental support at home to have successful outcomes with obesity prevention or management. Reducing the amount of sugar consumed by children can help decrease weight. Educating parents on how to modify the diet within the home and incorporate physical activity can have healthful benefits over time. Routinely weighing children and becoming accountable for modification of diet and incorporation of exercise can provide the needed support to children within the home. These resources provide support for home-based strategies in the prevention of pediatric obesity (Byrne et al., 2018 [23]; Nezami et al., 2018 [25]; Wright et al., 2018 [24]).

\section{Adult Obesity Prevention and Management}

\subsection{Contributing Factors to Obesity}

Prevention strategies, which include interventions, can help individuals who are at risk for obesity or already obese. Engaging in physical activity and modifying dietary choices can improve health and severity of chronic disease. Engaging the obese population in exercise can be a challenge, but necessary to improve health 
outcomes. Educating obese individuals who have chronic illness or are at risk for chronic illness is essential to prevent the progression of disease. Adults belonging to some ethnic groups are at higher risk of obesity than others. The studies reviewed the Latino and African American ethnic groups because these two groups of people are at high risk of obesity and need support. Healthy dietary options for these individuals can be a challenge as these individuals may have limited access to healthy foods and places to exercise. Providing educational opportunities on portion control and healthy eating as well as referrals to available resources to assist with increasing physical activity can be a benefit to these populations in order to prevent obesity (CDC, 2011 [7]; Hoelscher et al., 2015 [9]; Kisling \& Das, 2019 [8]).

\subsection{Managing Obesity Affiliated Chronic Diseases}

A self-management exercise program can help people with pre-hypertension and obesity (Tongyichean et al., 2019) [26]. There were thirty-two participants in this quasi-experimental study, who were diagnosed with pre-hypertension. Sixteen of the participants were part of the experimental group (self-managed exercise program), and the other sixteen were placed in the control group (conventional treatment). The results of the study showed that the experimental group had improved physical fitness compared to the control group and was able to improve in the following areas: cardiorespiratory fitness, muscular endurance, and flexibility. The study by Tongyichean et al. (2019) [26] supports obesity management in adults and provides evidence for overweight individuals with pre-hypertension who participate in a self-managed exercise program.

Using a commercial weight loss program can help individuals with pre-diabetes lose weight (Marrero et al., 2016) [27]. There were 225 people recruited to participate in this randomized control trial, who were diagnosed with pre-diabetes. One hundred and twelve participants followed a "Weight Watchers" plan compared to the 113 participants who followed the "Your Game Plan to Prevent Type 2 Diabetes" plan. The results showed that the "Weight Watchers" group lost a significantly larger amount of weight when compared to the "Your Game Plan to Prevent Type 2 Diabetes" group. The study by Marrero et al. (2016) [27], provides evidence supporting the use of "Weight Watchers" to help individuals with pre-diabetes lose weight and manage obesity.

Increasing physical activity and promoting self-regulation, which includes self-monitoring, goal setting, and time management can improve the behavioral effects of obese adults with type 2 diabetes (Silfee et al., 2016) [28]. This interventional study with randomization recruited participants by a few methods, including: researchmatch.com, flyers, or newsletters. A total of 23 participants enrolled for the pilot study and were older than 18 years of age.

Participants wore an armband during the first seven days (pre and post-test) to measure: steps taken daily and time of inactivity. The intervention and control group received information regarding the physical activity report; however, the 
intervention group received a pedometer, and the control group did not, the intervention group was given information on behavioral interventions including handouts to self-monitor, set goals, and manage time. Participants self-reported during the last four weeks regarding self-regulation strategies. The post-test results showed a significant improvement for the intervention group, as the intervention group had a better outcome in all areas when compared to the control group including self-regulation, self-monitoring, goal setting, social support, self-reward, time management, and overcoming barriers when compared to the control group. The study by Silfee et al. (2016) [28], provides evidence that gives support to individuals who are diagnosed with type 2 diabetes and obesity individuals will be better prepared to lose weight and manage obesity.

Another randomized control trial studies how mental health issues can lead to obesity (Jane et al., 2018) [29]. There were 137 participants who were recruited from the Perth community via advertisements. Weight was the primary intervention and the secondary interventions involved in the study included: energy intake and expenditure, psychological health, social relationships, physical health, quality of life, depression, anxiety, happiness, and opinions from the Facebook group. The participants were between the ages of 21 - 65 years of age and had a BMI between $25-40 \mathrm{~kg} / \mathrm{m}^{2}$. The participants were separated into three groups based on age in segments of 10 years, starting at age 21 . The standard group of participants followed the Australian government guidelines for dietary intake and the National Physical Activity Guidelines for Adults (Australian government). Participants had to attend clinical appointments at timed intervals over a 24-week period and bring a dietary journal and physical activity journal to monitor progress of the intervention. The other two groups were the pamphlet group and the Facebook group, whom both used the "total well-being diet," which is a low carbohydrate, high protein diet, and recommends walking 10,000 steps per day. The results showed that all groups lost weight; however, the Facebook group lost more weight and faster than the other two groups with a $p$ value of 0.016, when compared to the control group (Jane et al., 2018) [29]. The Facebook group also increased their psychological health score within the 12 -week period and the $\mathrm{p}$ value was 0.022 compared to the control group (Jane et al., 2018) [29]. This study by Jane et al. (2018) [29], is relevant to the topic of obesity management in obese adults, as it provided evidence supporting individuals who are looking for different methods to lose weight utilizing social media in particular Facebook.

Approximately two-thirds of adults in the United States are obese, and of that number, the majority are females (Shade et al., 2016) [30]. This trend is also affiliated with lack of sleep and health issues in overweight women who live in rural areas. This thirty-month randomized control trial recruited 301 middle-aged women who lived in rural areas. The study collected demographic information, anthropometric measures, biomarker variables, self-reported measurements of sleep disturbance as a result of pain, and a 24-hour sleep-wake study assessing 
sleep patterns at baseline, 6-months, and the change over time. The following information was obtained from the participants: age, menopausal status, height, weight, body mass index, waist circumference, blood pressure, and self-report of sleeping patterns. The results of this study showed that women who were consistently weighing-in over this time were able to improve weight, waist circumference, and blood pressure. The women who participated in the study were not able to improve sleep, as lack of sleep was related to the pain levels, and elevated blood pressure. This study by Shade et al. (2016) [30], provided evidence that supported mindfulness of weight and how that can improve outcomes when decreasing obesity, as evidenced by the participants losing weight and improving BMI.

There is a relationship between sleep disorders and obesity in the elderly (Taheri \& Irandoust, 2018) [31]. This intervention study with randomization had a sample of thirty-one participants who were 60 years old or older. The participants were screened using the Pittsburgh sleep quality index (PSQI) assessing BMI, waist/hip ratio, and percentage of body fat. The participants were separated into one of two groups: the aerobic exercise group and the group that did not exercise. The exercise group exercised for 60 minutes, three times per week, for two months. Results showed that quality of sleep improved for the exercise group, as well as BMI, waist/hip ratio, and percentage of body fat. This research by Taheri and Irandoust (2018) [31], provides evidence which supports adult obesity management, as evidenced by increasing physical activity and improved sleep quality.

Obesity related to chronic illness can be managed by the incorporation of diet and exercise. By utilizing these weight loss strategies, overall health can be improved as well as chronic illness management. The articles used on the topic of obesity management for individuals with chronic illness provide evidence supporting prevention strategies to manage obesity. Using Weight Watchers can help individuals lose weight by counting the amount of food taken in daily and benefit individuals diagnosed with pre-diabetes. Self-regulation can help individuals diagnosed with type 2 diabetes, and by providing tools to self-manage diabetes, obesity affiliated with this disease can be reduced. Individuals who have mental illness have shown an improvement in losing weight when using support systems such as Facebook. Sleep disorders were studied in relation to obesity, and increased activity was linked to improved sleeping habits. The results of these studies conclude that making the adult population responsible and accountable with self-regulation of diet and exercise can help improve outcomes related to weight loss. By introducing the information to adults regarding coping strategies or resources available and monitoring of chronic illness while introducing a change of diet and increasing physical activity (height, weight, BMI, waist circumference, lipid panels, and high blood pressure), people with chronic illness can successfully achieve weight loss or management. Using available resources for people with chronic illness such as; social media programs and cop- 
ing mechanisms can help manage weight and lead to successful outcomes of weight loss, and therefore diet and exercise can be used to manage obesity related to chronic illness (Jane et al., 2018 [29]; Marrero et al., 2016 [27]; Shade et al., 2016 [30]; Silfee et al., 2016 [28]; Taheri \& Irandoust, 2018 [31]; Tongyichean et al., 2019 [26]).

\subsection{Low-Income and Ethnicity}

Because Latinos are at higher risk of developing chronic disease related to obesity, there is a need to research if an obesity prevention program would work in the community (Cotter et al., 2018) [32]. Socio-economic status does influence dietary choices for individuals in this population. This study was a qualitative-descriptive study, and some general methods used in phase one of the study involved recruiting 21 Latinos to assess dietary needs and interests. In phase two, there were 41 Latinos selected in the process of intervention, which involved: mindful eating, and behavioral methods to manage stress. The author stated that there were challenges of consistent participation of the participants due to work or family. Future considerations included possible payment to increase interest, contracts at the beginning, and more follow-up. The results of this study concluded that most of the participants would be willing to change dietary habits because of participating in mindful eating and behavioral methods to manage stress. This study by Cotter et al. (2018) [32] showed moderate evidence supporting a need for Latinos to become mindful of eating habits to prevent obesity.

Latino women are at high risk for cardiovascular disease related to obesity prevalence (Koniak-Griffin et al., 2015) [33]. The participants who were recruited for this randomized controlled trial included 223 women between the ages of 35 - 64 years of age with an education level of eight grade or less and are also from a low-socioeconomic status. The intervention group attended eight classes on lifestyle changes with four months of coaching via phone or home visits following. The control group received training regarding topics not related to healthy lifestyle changes. After the nine-month study concluded, the results showed that providing classes for this population can be a benefit in the prevention of cardiovascular disease related to obesity. The intervention group showed an increase in time spent engaging in physical activity when compared to the control group. The intervention group was able to decrease BMI and waist circumference as well, which provided for increased knowledge regarding cardiovascular disease, as well as improving dietary habits, increasing the number of steps, and improving waist circumference. This study provides evidence supporting a need for lifestyle changes to manage and control obesity to prevent complications related to chronic disease.

The risk of developing chronic disease in the African American population can be decreased by preventing obesity (Burton et al., 2017) [34]. A systematic review of studies from 2005-2015 was conducted, which included 147 sources from several databases, including: Nursing and Allied Health Plus with Full 
Text, Academic Search Premier, Education Resources Information Center, Medline, and Cochrane. Burton et al. (2017) [34] reduced the search to eight articles involving an intervention for obesity in the African American population during that time. The eight studies focused on weight loss by increasing the amount of fruits and vegetables consumed, as well as increasing activity level. There were some concerns by Burton et al. (2017) [34], regarding the ability to meet the goals of these interventions and the following was mentioned: access to healthy affordable foods, and areas to promote increased physical activity need to be considered when developing a plan for this population. The results of the study showed evidence of weight reduction in tailoring obesity interventions for this population. This systematic review looked at studies over ten years related to the topic of weight reduction interventions for the African American population. This research provides support to increase awareness of nutrition and increased physical activity to meet the needs of this ethnic population. The evidence provided in this research study supports weight reduction interventions that are tailored to the individual needs of the African American population.

Increasing physical activity and improving dietary choices can prevent the African American population from developing chronic illnesses (Halbert et al., 2017) [35]. The research design used for this study was quasi-experimental design, which was randomized. Sampling consisted of a needs assessment of African Americans living in Philadelphia, Pennsylvania, and found that individuals were concerned with chronic diseases associated with obesity.

Participants were African American individuals between the ages of 18 - 75 and resided in Philadelphia's metropolitan areas. The results of this study showed that educating this population regarding the risks of chronic disease related to lifestyle choices elicited a positive response to change amongst the participants who were educated. The study provides moderate evidence supporting education for diverse adult populations regarding the risks of unhealthy living and the benefits of preventing obesity.

Offering a behavioral weight-loss intervention can work to help underserved African American people who are overweight or obese in underserved urban areas (Joshi et al., 2018) [36]. The study is a randomized control trial which involved recruiting African American women from the Boston Medical Center to join the Revolutionizing Exercise and Nutrition Every day in Women (RENEW). There were two groups of participants, one group was the intervention group, and the other group was the usual care group. The results showed that the participant satisfaction rate was higher in the intervention group than in the usual care group. Both groups of women, however, showed an increase in weight. Some concluding thoughts were that the participants would need to attend more of the program sessions in order to lose weight. The study was considered a success, as participants were satisfied with the program. This study is relevant to the management of obesity as becoming involved in a program that supports exercise and nutrition can have positive outcomes if participants are compliant. This 
article by Joshi et al. (2018) [36], provides evidence supporting a need for programs to help underserved African American people.

Generally, the research supports a need for education for the diverse populations, which include the African American and Latino populations. These studies provide evidence for individuals to incorporate healthy lifestyle choices and opportunities to prevent obesity and other chronic illnesses. Latinos are at high risk for obesity and can benefit from improving dietary choices. The African American population pose a high risk for chronic disease, which can be prevented by reducing weight and modifying lifestyle choices. These articles support a need for education for the diverse populations which included the African American and Latino populations (Burton et al., 2017 [34]; Cotter et al., 2018 [32]; Halbert et al., 2017 [35]; Joshi et al., 2018 [36]; Koniak-Griffin et al., 2015 [33]).

\subsection{Physical Activity}

There are many options available to adults to utilize daily to prevent obesity. Making adults aware of available resources is important to have positive outcomes and prevent obesity and obesity-related chronic diseases. Educating adults regarding an adequate amount of physical exercise, and dietary consumption recommendations can elicit a change for health promotion.

Using available technology to track steps taken can support weight management and loss. In this section, articles review obesity prevention strategies for adults by improving nutritional intake and increasing physical activity (Harada et al., 2018 [5]; Hoelscher et al., 2015 [9]). Interventions can also help individuals who are already obese. Engaging in physical activity and modifying dietary choices can improve health and severity of chronic disease. Engaging the obese population in exercise can be a challenge, but necessary to improve health outcomes. Providing healthy options for obese individuals, or those who are at risk for obesity can lead to successful healthy outcomes and improvement of health (CDC, 2020 [1]; NIH, 2020 [4]; Mayo Clinic, 2015).

It is recommended by the world health organization (WHO) that an adult should spend 150 minutes per week in moderate physical activity or 75 minutes per week of vigorous physical activity to promote health and prevent obesity (Harada et al., 2019) [5]. This experimental study included 262 participants, who were adults scoring between a $21-24$ on the mini-mental state examination (MMSE). This study looked at physical activity level, self-efficacy, self-regulation, and environmental factors. The results of this study showed that physical activity level and self-efficacy are related to the older adults with cognitive decline and self-regulation for which environmental factors were not related to the effects of physical activity in this population. This study is relevant to the topic of obesity prevention in adults and provides evidence supporting the need for increased physical activity in adults, which is related to self-efficacy for adults with cognitive decline (Harada et al., 2019) [5].

Healthcare can change the focus from obesity treatment to weight gain pre- 
vention (Metzgar \&Richardson, 2016) [37]. This randomized controlled trial lasted one year and included premenopausal women who were between the ages of 18 - 45. There were 87 women in this study. The intervention group was seen by a registered dietician, and the control group was seen by a counselor. Baseline information was collected and included: anthropometric measures, blood pressure, dietary intake, physical activity level, and biochemical markers, which were obtained every three months. The results of the study showed that all women were able to maintain weight regardless of the group. There was a lower percentage of body fat in the intervention group compared to the control group. This study by Metzgar and Richardson (2016) [37] is an excellent source to prevent weight gain in women and provides evidence supporting monitoring of dietary intake, physical activity level, blood pressure, anthropometric measures, and biochemical markers at regular intervals which can lead to successful weight management and prevention of adult obesity.

\subsection{Motivation and Self-Regulation}

Applying the motivation theory can help women change attitudes toward increasing physical activity (Mirkarimi et al., 2017) [38]. This randomized control trial evaluated sixty overweight women. The participants were separated into two groups, one with a standardized weight-control program and the other with motivational interviewing. Height, weight, body mass index, and waist circumference measurements were obtained on all sixty participants. The results of the study revealed that the group of women in the motivational group was at an increased benefit showing an increase in physical endurance and an increased amount of weight loss compared to the control group or standardized weight-controlled group. The study by Mirkarimi et al. (2017) [38], provides evidence supporting physical endurance to activity and an increased amount of weight loss, therefore managing obesity in overweight adults.

Self-regulation can help young adults reduce the chance of weight gain over time (Wing et al., 2016) [39]. This randomized clinical trial enrolled 600 participants between the ages of 18 - 35 with a body mass index between 21 - 30.9 (normal and obese ranges). The control group met one time compared to the two intervention groups (small change and large change) that met ten times over four months. Weight was obtained two times during the study period of 4 months and then again after three years. The intervention groups were able to maintain weight successfully, and the study concluded that the smaller change intervention over time was most successful at maintaining weight and prevention of obesity. This study by Wing et al. (2016) [39] provides evidence that supports educating people on lifestyle changes that can influence self-regulation and weight management.

When evaluating the resources for adult obesity prevention and management of interventions or treatments, there are many considerations to improve outcomes. Spending 150 hours engaged in moderate physical activity every week 
can help the older adult population with cognitive decline, prevent obesity. Weight gain prevention strategies can help adult women and prevent obesity by increasing physical activity and improving dietary intake. Self- regulation regarding diet and exercise can manage obesity and prevent chronic diseases. Increasing physical engagement by motivating the female population can reduce weight and manage obesity. These articles provide evidence that supports adult obesity prevention interventions and have positive outcomes in the prevention of chronic disease associated with obesity (Harada et al., 2019 [5]; Metzgar \& Richardson, 2016 [37]; Mirkarimi et al., 2017 [38]; Wing et al., 2016 [39]).

\subsection{Nutrition}

Sugar consumption can cause an increased risk of obesity, coronary heart disease, diabetes, metabolic syndrome, nonalcoholic fatty liver disease, and brain stimulation, which increases the need for sugar (Rippe \& Angelopoulos, 2015) [40]. The methods of reviewing data in this systematic review involved looking at 30 randomized control trials and 38 prospective studies. These studies looked at how increasing sugar consumption affected the body. The following effects were reviewed: blood pressure, lipids, diabetes, and liver fat accumulation. The results of the study concluded that increasing sugar intake and decreasing physical activity places one at risk for coronary heart disease, diabetes, metabolic syndrome, and fatty liver disease. This study provides evidence supporting the need to educate individuals regarding the risk of chronic diseases related to obesity caused by increased sugar consumption.

Being weighed on a regular basis can help prevent weight gain (Rosenbaum et al., 2017) [41]. This longitudinal study, which lasted for two years, involved 294 young women ages eighteen to nineteen years of age. The following items were measured: BMI, body composition (using a dexa scan), and self-weighing frequency a data questionnaire was collected prior to the study to establish a baseline. Participants were expected to weigh themselves at least one time per week over two years. The results showed that from the sixth-month mark into the study to two years that the body fat percentage decreased in the participants who weighed themselves regularly.

This study is an excellent resource that provides evidence that individuals who are regularly weighed can manage weight proactively.

\subsection{Nutritional Supplements}

There are benefits of consuming extra virgin olive oil in the reduction of obesity by reducing excess body fat (Galvao et al., 2019) [42]. This randomized controlled trial recruited 41 women. The control group had 20 women who were given high-fat breakfasts made with soybean oil, and the intervention group had the remaining 21 women who received the same breakfast made with extra virgin olive oil. Anthropometric measures were assessed on the first and last day, as well as lab studies, and dual-energy X-Ray absorptiometry. The results showed 
that fat loss was approximately $80 \%$ higher in the intervention group than in the control group, as well as a decrease in diastolic blood pressure. The study by Galvao et al. (2019) [42], provides evidence that extra virgin olive oil can help reduce fat and help in the management of obesity.

Green tea is an herbal supplement that can help obese adults reduce body weight, therefore, decreasing the associated risks of obesity (Singh \& Kumar, 2019) [43]. This quasi-experimental study reviewed how green tea affected body weight. The sample size consisted of 60 adult females between the ages of $20-35$ years of age. Demographic information was obtained from participants, such as: age, religion, educational status, monthly family income, dietary pattern, and body mass index. Results of the study showed that green tea is effective in reducing weight and managing obesity. This study is relevant to the topic of obesity prevention and management, as there is evidence supporting obesity prevention by using green tea to supplement the diet.

Probiotics can play a role in controlling obesity, improve metabolism, and decrease inflammation (Wang et al., 2019) [44]. This systematic review of the literature involved three databases that searched randomized control trials. A total of 1255 studies were generated from the three databases that matched the search, and 12 studies matched the search criteria. The studies included 821 participants, who were given probiotics in different forms, such as: capsule, yogurt, sachet, powder, or fermented milk. The participants who received the probiotics showed a loss of weight compared to the participants who did not take probiotics. This study provides evidence that probiotics can help prevent and manage obesity.

When evaluating the resources regarding nutrition, these articles provide evidence to support obesity prevention and management in adults by improving nutritional intake.

Decreasing sugar intake can help reduce the risk of acquiring chronic diseases. Having adults take responsibility for self-regulation and weighing themselves on a regular basis can lead to decreased caloric intake and increased physical activity. The resources regarding nutritional supplements provide evidence supporting the use of virgin olive oil, green tea, and probiotics to reduce fat. These supplements were used by individuals within these studies, which concluded that all the supplements have a positive affiliation with the reduction of fat and body weight. The use of the mentioned supplements could be incorporated into the daily diet for positive results. The research on nutritional supplementation as an additive to the diet can benefit overweight or obese individuals (Galvao et al., 2019 [42]; Singh \& Kumar, 2019 [43]; Rippe \& Angelopoulos, 2015 [40]; Rosenbaum et al., 2017 [41]; Wang et al., 2019 [44]).

\subsection{Use of Technology}

Social media can prove to be a resource for weight behavior interventions (Ruopeng et al., 2017) [45]. This systematic review with a meta-analysis conducted a search in the Cochrane Library database to retrieve twenty-two interventions, 
including: twelve randomized controlled trials, six pre/post studies, and three cohort studies from nine different countries. Intervention duration averaged 17.8 weeks, and the average sample size of participants was sixty-nine. The study showed an improvement when using social media-based interventions for weight loss.

Results showed that BMI, waist circumference, and body weight were all decreased, while the number of steps taken within a day was increased. This research by Ruopeng et al. (2017) [45], is an excellent resource for obesity prevention in the adult population by increasing daily steps and provides evidence supporting the topic of obesity prevention in the adult population using the social media platform.

Using mobile technology can prevent obesity by providing adults with information regarding physical activity, sedentary behavior, and sleep (Elavsky et al., 2019) [46]. This systematic literature review used eight databases to gather information regarding mobile health intervention. The participants of the study included adults aged 50 and older and targeted the topic of physical activity, sedentary behavior, and sleep. Studies involving interventions that were focused on Health, such as: telemedicine, telemonitoring, telephone calls, exergames, or use of health-based websites, were reviewed. The search retrieved 2260 articles, of which 52 were used to conduct this research. The mean number of participants in the 52 articles was 114, and the mean age of participants was 59.6 years of age. Thirty-three of the articles focused on physical activity, thirteen of the articles focused on both physical activity and sedentary lifestyle. The study concluded that there is evidence that mobile health technology can improve physical activity and decrease sedentary lifestyle for adults who are 50 years old and older. This article provides evidence supporting the use of technology to increase physical activity in adults over the age of fifty (Elavsky et al., 2019) [46].

These resources provide evidence supporting the use of technology to lose weight. The use of social media had positive results regarding individual engagement in physical activity. Individuals were able to record the number of steps taken daily and had the needed support from other participants on social media sites. Older individuals were able to benefit from using mobile technology to improve physical activity and lead a more active life. The use of technology can be a helpful tool for adults to use daily and provide convenience availability and support (Elavsky et al., 2019 [46]; Ruopeng et al., 2017 [45]).

\section{Conclusions}

In conclusion, the resources used in researching the topic of obesity prevention and management throughout the lifespan provide a variety of options for all ages to utilize to achieve positive outcomes. By offering options for individuals to change lifestyle, chronic disease can be prevented or managed. Healthcare providers and educators can teach individuals how to eat healthier and refer to community programs that offer physical activity programs. This review studied 
several resources on the topic of obesity prevention or management for the pediatric population, which includes adolescents, and adult populations.

The resources found for the pediatric and adolescent populations provided evidence that prevention strategies can be used to prevent obesity in this population. When reviewing resources regarding contributing factors, which include chronic illnesses, socioeconomic status, and ethnic considerations, the pediatric population can have challenges to overcome to prevent or manage obesity. Providing support in the home and community is needed to ensure that chronic diseases that are affiliated with obesity are prevented in the future (Cloutier et al., 2018 [10]; French et al., 2018 [11]; Gatto et al., 2017 [12]; Hull et al., 2018 [13]; Lofton et al., 2016 [14]; NIH, 2020 [4]; Papoutsakis et al., 2018 [19]; Rubin et al., 2018 [18]; Smith et al., 2016 [15]; Trude et al., 2018 [16]; Woodward et al., 2018 [17]).

When reviewing the resources on the topic of nutrition and physical activity prevention strategies in the pediatric population, evidence is provided supporting involvement from the community, which includes the school-setting, physician's office, and home. Children can utilize games to learn how to use portion control and physical activity. Parental involvement with lifestyle changes in the home is essential to have successful outcomes with obesity prevention and management. Learning how to prepare healthy meals and parental support for increased physical activity in the home can elicit positive outcomes in prevention and management of pediatric obesity (Byrne et al., 2018 [23]; Nezami et al., 2018 [25]; Staiano et al., 2018 [22]; Viggiano et al., 2018 [21]; Wright et al., 2018 [24]).

In regard to the adult population, contributing factors involving chronic illness or risk for chronic illnesses such as: sleep disorders, pre-hypertension, pre-diabetes, and type 2 diabetes showed evidence supporting modification of lifestyle by incorporating self-regulation or management strategies including increased physical activity and modification of diet to improve health. The low-income and ethnic populations were also studied involving the Hispanic and African American populations and how these populations have limited opportunities for activity, due to urban living, and access to healthy food which is related to cost and durability of healthy food (Burton et al., 2017 [34]; Cotter et al., 2018 [32]; Halbert et al., 2017 [35]; Jane et al., 2018 [29]; Joshi et al., 2018 [36]; Koniak-Griffin et al., 2015 [33]; Marrero et al., 2016 [27]; Shade et al., 2016 [30]; Silfee et al., 2016 [28]; Taheri \& Irandoust, 2018 [31]; Tongyichean et al., 2019 [26]).

Other aspects of obesity in the adult population that were reviewed were physical activity and nutrition and how individuals can utilize making changes to prevent or manage obesity.

These articles reviewed the recommended amount of physical activity, nutrition, and technology that individuals can use to track steps taken in a day, and technology that can add caloric intake of food. These resources can benefit someone who is at risk of becoming obese or already obese and manage weight 
effectively. These articles provide alternatives or supplementation to diet and exercise to assist anyone, who has access to nutritional supplements, or online applications to manage weight (Elavsky et al., 2019 [46]; Galvao et al., 2019 [42]; Harada et al., 2019 [5]; Metzgar \& Richardson, 2016 [37]; Mirkarimi et al., 2017 [38]; Rippe \& Angelopoulos, 2015 [40]; Rosenbaum et al., 2017 [41]; Ruopeng et al., 2017 [45]; Singh \& Kumar, 2019 [43]; Wang et al., 2019 [44]; Wing et al., 2016 [39]).

The content from all the research articles included ways for individuals to reduce obesity by incorporating increased physical activity and modification of diet. The research articles used to develop this literature review provided evidence supporting increased physical activity and eating a healthy diet to decrease the risk of obesity and chronic illnesses affiliated with obesity. This applies to the field of nursing as preventing or managing obesity is vital to help patients of all ages improve health and live longer lives.

Future directions of this research could lead to taking one of these topics within this review, such as low-socioeconomic status and focusing on that topic. As a healthcare worker and educator in the state of West Virginia, there are many reasons that people have limited access to healthy foods. Poverty is one reason, rural living, and availability of healthy foods.

West Virginia is the second most populated state in the United States with individuals who are diagnosed with type 2 diabetes. There is a need to educate these individuals regarding healthy options for eating daily, as well as options for increased activity.

New directions of the research are looking at barriers to health promotion activities for nursing students while in nursing school. Due to the rigor of the course work in nursing school, nursing students do not engage in healthy activities such as good nutrition, and physical activity as time is spent engaging in academics for successful outcomes. The nursing student must prepare for lectured material in the classroom, skills training in the lab, and clinical practice to learn care on actual patients. The student is responsible for preparing to test, learn skills, practice skill, test on skills, preplan, and patient care. Due to limited time and increased stress, students need support to maintain health and wellness in nursing school and into the profession of nursing. Role modeling healthy behaviors is important as nursing students and nurses educate patients on health promotion to prevent or maintain disease (Bryer et al., 2013) [47].

\section{Conflicts of Interest}

The author declares no conflicts of interest regarding the publication of this paper.

\section{References}

[1] Centers for Disease Control and Prevention (2020) Adult Obesity Facts. https://www.cdc.gov/obesity/data/adult.html

[2] Mayo Clinic (2020) Obesity. 
https://www.mayoclinic.org/diseases-conditions/obesity/symptoms-causes/syc-2037 $\underline{5742}$

[3] National Institutes of Health (2015) Health Risks of Being Overweight. https://www.niddk.nih.gov/health-information/weight-management/health-risks-o verweight

[4] National Institutes of Health (2020) Prader-Willi Syndrome. (Childhood Obesity Facts, 2019) https://ghr.nlm.nih.gov/condition/prader-willi-syndrome

[5] Harada, K., Lee, S., Lee, S., Bae, S., Harada, K., Suzuki, T. and Shimada, H. (2019) Psychological and Environmental Correlates of Moderate-to-Vigorous Physical Activity and Step Counts among Older Adults with Cognitive Decline. Perceptual \& Motor Skills, 126, 639-655. https://doi.org/10.1177/0031512519846026

[6] MyPlate Partner Title Become a MyPlate Partner! (n.d.). https://www.choosemyplate.gov/

[7] Centers for Disease Control and Prevention (2011) State Smoke-Free Laws for Worksites, Restaurants, and Bars-United States, 2000-2010. PsycEXTRA Dataset. https://www.cdc.gov/pictureofamerica/pdfs/picture_of_america_prevention.pdf

[8] Kisling, L.A. and Das, J.M. (2019) Prevention Strategies. StatPearls Publishing. https://www.ncbi.nlm.nih.gov/books/NBK537222/

[9] Hoelscher, D.M., Butte, N.F., Barlow, S., Vandewater, E.A., Sharma, S.V., Huang, T., Finkelstein, E., Pont, S., Sacher, P., Byrd-Williams, C., Oluyomi, A.O., Durand, C., Li, L. and Kelder, S.H. (2015) Incorporating Primary and Secondary Prevention Approaches to Address Childhood Obesity Prevention and Treatment in a Low-Income, Ethnically Diverse Population: Study Design and Demographic Data from the Texas Childhood Obesity Research Demonstration (TX CORD) Study. Childhood Obesity (Print), 11, 71-91. https://doi.org/10.1089/chi.2014.0084

[10] Cloutier, M.M., Wiley, J.F., Kuo, C.L., Cornelius, T., Wang, Z. and Gorin, A.A. (2018) Outcomes of an Early Childhood Obesity Prevention Program in a Low-Income Community: A Pilot, Randomized Trial. Pediatric Obesity, 13, 677-685. https://doi.org/10.1111/ijpo.12458

[11] French, S.A., Sherwood, N.E., Veblen-Mortenson, S., Crain, A.L., JaKa, M.M., Mitchell, N.R., Hotop, A.M., Berge, J.M., Kunin Batson, A.S., Truesdale, K., Stevens, J., Pratt, C. and Esposito, L. (2018) Multicomponent Obesity Prevention Intervention in Low-Income Preschoolers: Primary and Subgroup Analyses of the NET-Works Randomized Clinical Trial, 2012-2017. American Journal of Public Health, 108, 1695-1706. https://doi.org/10.2105/AJPH.2018.304696

[12] Gatto, N.M., Martinez, L.C., Spruijt, M.D. and Davis, J. N. (2017) LA Sprouts Randomized Controlled Nutrition, Cooking and Gardening Programme Reduces Obesity and Metabolic Risk in Hispanic/Latino Youth. Pediatric Obesity, 28-37. https://doi.org/10.1111/ijpo.12102

[13] Hull, P.C., Buchowski, M., Canedo, J.R., Beech, B.M., Du, L., Koyama, T. and Zoorob, R. (2018) Childhood Obesity Prevention Cluster Randomized Trial for Hispanic Families: Outcomes of the Healthy Families Study. Pediatric Obesity, 13, 686-696. https://doi.org/10.1111/ijpo.12197

[14] Lofton, S., McNaughton, D.B., Julion, W.A., Bergren, M.D. and Keim, K.S. (2016) A Systematic Review of Literature on Culturally Adapted Obesity Prevention Interventions for African American Youth. Journal of School Nursing, 32, 32-46. https://doi.org/10.1177/1059840515605508

[15] Smith, J.J., Morgan, P.J., Plotnikoff, R.C., Stodden, D.F. and Lubans, D.R. (2016) Mediating Effects of Resistance Training Skill Competency on Health-Related Fit- 
ness and Physical Activity: The ATLAS Cluster Randomised Controlled Trial. Journal of Sports Sciences, 34, 772-779. https://doi.org/10.1080/02640414.2015.1069383

[16] Trude, A.C.B., Surkan, P.J., Cheskin, L.J. and Gittelsohn, J. (2018) A Multilevel, Multicomponent Childhood Obesity Prevention Group-Randomized Controlled Trial Improves Healthier Food Purchasing and Reduces Sweet-Snack Consumption among Low-Income African American Youth. Nutritional Journal, 17, Article No.: 96. https://nutritionj.biomedcentral.com/articles/10.1186/s12937-018-0406-2 https://doi.org/10.1186/s12937-018-0406-2

[17] Woodward, L.G., Gosliner, W., Au, L.E., Kao, J., Webb, K.L., Sagatov, R.D., Strauss, W.J., Landgraf, A.J., Nagaraja, J., Wilson, D.K., Nicastro, H.L., Nebeling, L.C., Schultz, J.A. and Ritchie, L.D. (2018) Community Characteristics Modify the Relationship between Obesity Prevention Efforts and Dietary Intake in Children: The Healthy Communities Study. Pediatric Obesity, 13, 46-55.

https://doi.org/10.1111/ijpo.12434

[18] Rubin, D., Wilson, K.S., Honea, K.E., Castner, D.M., McGarrah, J.G., Rose, D.J. and Dumont-Driscoll, M. (2019) An Evaluation of the Implementation of a Parent-Led, Games-Based Physical Activity Intervention: The Active Play at Home Quasi-Randomized Trial. Health Education Research, 34, 98-112. https://doi.org/10.1093/her/cyy035

[19] Papoutsakis, C., Papadakou, E., Chondronikola, M., Antonogeorgos, G., Matziou, V., Drakouli, M., Konstantaki, E. and Priftis, K.N. (2018) An Obesity-Preventive Lifestyle Score Is Negatively Associated with Pediatric Asthma. European Journal of Nutrition, 57, 1605-1613. https://doi.org/10.1007/s00394-017-1446-7

[20] Centers for Disease Control and Prevention (2019) Childhood Obesity Facts. https://www.cdc.gov/obesity/data/childhood.html

[21] Viggiano, E., Viggiano, A., Di Costanzo, A., Viggiano, A., Viggiano, A., Andreozzi, E., Romano, V., Vicidomini, C., Di Tuoro, D., Gargano, G., Incarnato, L., Fevola, C., Volta, P., Tolomeo, C., Giuseppina, S., Santangelo, C., Apicella, M., Battista, R., Raia, M., Amaro, S., et al. (2018) Healthy Lifestyle Promotion in Primary Schools through the Board Game Kaledo: A Pilot Cluster Randomized Trial. European Journal of Pediatrics, 177, 1371-1375. https://doi.org/10.1007/s00431-018-3091-4

[22] Staiano, A.E., Beyl, R.A., Guan, W., Hendrick, C.A., Hsia, D.S. and Newton, R.L. (2018) Home-Based Exergaming among Children with Overweight and Obesity: A Randomized Clinical Trial. Pediatric Obesity, 13, 724-733.

https://doi.org/10.1111/ijpo.12438

[23] Byrne, J.L.S., Browne, N.E., Cameron Wild, T., Maximova, K., Holt, N.L., Cave, A. J., Martz, P., Ellendt, C. and Ball, G.D.C. (2018) A Brief eHealth Tool Delivered in Primary Care to Help Parents Prevent Childhood Obesity: A Randomized Controlled Trial. Pediatric Obesity, 13, 659-667. https://doi.org/10.1111/ijpo.12200

[24] Nezami, B.T., Ward, D.S., Tate, D.F., Ennett, S.T. and Lytle, L.A. (2018) A mHealth Randomized Controlled Trial to Reduce Sugar-Sweetened Beverage Intake in Preschool-Aged Children. Pediatric Obesity, 13, 668-676.

https://doi.org/10.1111/ijpo.12258

[25] Wright, J.A., Whiteley, J.A., Watson, B.L., Gorin, S.N.S. and Hayman, L.L. (2018) Tailored Communications for Obesity Prevention in Pediatric Primary Care: A Feasibility Study. Health Education Research, 33, 14-25. https://doi.org/10.1093/her/cyx063

[26] Tongyichean, T., Aungsuroch, Y. and Preechawong, S. (2019) Effect of Self-Management Exercise Program on Physical Fitness among People with Pre-Hypertension and Obesity: A Quasi-Experimental Study. Pacific Rim International Journal of Nursing Re- 
search, 23, 6-17. https://he02.tci-thaijo.org/index.php/PRIJNR/article/view/108336

[27] Marrero, D.G., Palmer, K.N.B., Phillips, E.O., Miller-Kovach, K., Foster, G.D. and Saha, C.K. (2016) Comparison of Commercial and Self-Initiated Weight Loss Programs in People with Pre-Diabetes: A Randomized Control Trial. American Journal of Public Health, 106, 949-956. https://doi.org/10.2105/AJPH.2015.303035

[28] Silfee, V., Petosa, R., Laurent, D., Schaub, T. and Focht, B. (2016) Effect of a Behavioral Intervention on Dimensions of Self-Regulation and Physical Activity among Overweight and Obese Adults with Type 2 Diabetes: A Pilot Study. Psychology, Health \& Medicine, 21, 715-723. https://doi.org/10.1080/13548506.2016.1139144

[29] Jane, M., Foster, J., Hagger, M., Ho, S., Kane, R. and Pal, S. (2018) Psychological Effects of Belonging to a Facebook Weight Management Group in Overweight and Obese Adults: Results of a Randomised Controlled Trial. Health \& Social Care in the Community, 26, 714-724. https://doi.org/10.1111/hsc.12584

[30] Shade, M.Y., Berger, A.M., Dizona, P.J., Pozehl, B.J. and Pullen, C.H. (2016) Sleep and Health-Related Factors in Overweight and Obese Rural Women in a Randomized Controlled Trial. Journal of Behavioral Medicine, 39, 386-397. https://doi.org/10.1007/s10865-015-9701-y

[31] Taheri, M. and Irandoust, K. (2018) The Exercise-Induced Weight Loss Improves Self-Reported Quality of Sleep in Obese Elderly Women with Sleep Disorders. Sleep \& Hypnosis, 20, 54-59. https://doi.org/10.5350/Sleep.Hypn.2017.19.0134

[32] Cotter, E., Bera, V., Elsemore, J. and Sneling, A. (2018) Examining the Feasibility and Effectiveness of a Community-Based Obesity Prevention Program. American Journal of Health Education, 49, 94-104. https://doi.org/10.1080/19325037.2017.1414643

[33] Koniak-Griffin, D., Brecht, M.-L., Takayanagi, S., Villegas, J., Melendrez, M. and Balcazar, H. (2015) A Community Health Worker-Led Lifestyle Behavior Intervention for Latina (Hispanic) Women: Feasibility and Outcomes of a Randomized Controlled Trial. International Journal of Nursing Studies, 52, 75-87. https://doi.org/10.1016/j.ijnurstu.2014.09.005

[34] Burton, W.M., White, A.N. and Knowlden, A.P. (2017) A Systematic Review of Culturally Tailored Obesity Interventions among African American Adults. American Journal of Health Education, 48, 185-197. https://doi.org/10.1080/19325037.2017.1292876

[35] Halbert, C.H., Bellamy, S., Briggs, V., Delmoor, E., Purnell, J., Rogers, R., Weathers, B. and Johnson, J.C. (2017) A Comparative Effectiveness Education Trial for Lifestyle Health Behavior Change in African Americans. Health Education Research, 32, 207-218. https://doi.org/10.1093/her/cyx039

[36] Joshi, P.P., Quintiliani, L.M., McCarthy, A.C., Gilmore, A., Mahesri, M., Sullivan, L. M. and Apovian, C.M. (2018) A Randomized Controlled Feasibility Trial in Behavioral Weight Management for Underserved Postpartum African American Women: The RENEW Study. Preventing Chronic Disease, 15, 1-4. https://doi.org/10.5888/pcd15.170400

[37] Metzgar, C.J. and Nickols-Richardson, S.M. (2016) Effects of Nutrition Education on Weight Gain Prevention: A Randomized Controlled Trial. Nutrition Journal, 15, 1-13. https://doi.org/10.1186/s12937-016-0150-4

[38] Mirkarimi, K., Eri, M., Ghanbari, M.R., Kabir, M.J., Raeisi, M., Ozouni-Davaji, R., B., Aryaie, M. and Charkazi, A. (2017) Modifying Attitude and Intention toward Regular Physical Activity Using Protection Motivation Theory: A Randomized Controlled Trial. Easter Mediterranean Health Journal, 23, 543-550. 
https://doi.org/10.26719/2017.23.8.543

[39] Wing, R.R., Tate, D.F., Espeland, M.A., Lewis, C.E., LaRose, J.G., Gorin, A.A., Bahnson, J., Perdue, L.H., Hatley, K.E., Ferguson, E., Garcia, K.R. and Lang, W. (2016) Innovative Self-Regulation Strategies to Reduce Weight Gain in Young Adults: The Study of Novel Approaches to Weight Gain Prevention (SNAP) Randomized Clinical Trial. JAMA Internal Medicine, 176, 755-762.

https://doi.org/10.1001/jamainternmed.2016.1236

[40] Rippe, J.M. and Angelopoulos, T.J. (2015) Sugars, Obesity, and Cardiovascular Disease: Results from Recent Randomized Control Trials. European Journal of Nutrition, 55, 45-53. https://doi.org/10.1007/s00394-016-1257-2

[41] Rosenbaum, D., Espel, H., Butryn, M., Zhang, F. and Lowe, M. (2017) Daily Self-Weighing and Weight Gain Prevention: A Longitudinal Study of College-Aged Women. Journal of Behavioral Medicine, 40, 846-853. https://doi.org/10.1007/s10865-017-9870-y

[42] Galvão Cândido, F., Xavier Valente, F., da Silva, L.E., Gonçalves Leão Coelho, O., Gouveia Peluzio, M.C. and Gonçalves Alfenas, R.C. (2018) Consumption of Extra Virgin Olive Oil Improves Body Composition and Blood Pressure in Women with Excess Body Fat: A Randomized, Double-Blinded, Placebo-Controlled Clinical Trial. European Journal of Nutrition, 57, 2445-2455.

https://doi.org/10.1007/s00394-017-1517-9

[43] Singh, R. and Kumar, R.S.R. (2019) A Study to Assess the Effectiveness of Green Tea on Reduction of Body Weight among Obese Young Adult Girls Residing at Selected Girl's Hostel of Sharda University, Greater Noida. International Journal of Nursing Education, 11, 62-66.

[44] Wang, Z.-B., Xin, S.-S., Ding, L.-N., Ding, W.-Y., Hou, Y.-L., Liu, C.-Q. and Zhang, X.-D. (2019) The Potential Role of Probiotics in Controlling Overweight/Obesity and Associated Metabolic Parameters in Adults: A Systematic Review and Meta-Analysis. Evidence-Based Complementary \& Alternative Medicine (ECAM), 2019, Article ID: 3862971. https://doi.org/10.1155/2019/3862971

[45] An, R.P., Ji, M.M. and Zhang, S. (2017) Effectiveness of Social Media-Based Interventions on Weight-Related Behaviors and Body Weight Status: Review and Metaanalysis. American Journal of Health Behavior, 41, 670-682. https://doi.org/10.5993/AJHB.41.6.1

[46] Elavsky, S., Knapova, L., Klocek, A. and Smahel, D. (2019) Mobile Health Interventions for Physical Activity, Sedentary Behavior, and Sleep in Adults Aged 50 Years and Older: A Systematic Literature Review. Journal of Aging \& Physical Activity, 27, 565-593. https://doi.org/10.1123/japa.2017-0410

[47] Bryer, J., Cherkis, F. and Raman, J. (2013) Health-Promotion Behaviors of Undergraduate Nursing Students: A Survey Analysis. Nursing Education Perspectives, 34, 410-415. https://doi.org/10.5480/11-614 